\title{
Filmförderung als Transformationsbremse. Die politische Gestaltung der Kinospielfilmproduktion in Deutschland
}

\section{Thomas Wiedemann}

Keywords: Medienpolitik, Medienproduktion, Spielfilm, Akteur-StrukturDynamiken, Bedeutungskonstitution

\section{Abstract}

Unter Bezugnahme auf Schimanks Ansatz der Akteur-Struktur-Dynamiken fragt der Beitrag kritisch nach den Mechanismen, die das deutsche Filmschaffen angesichts des filmpolitischen Gestaltungswillens hierzulande prägen. Auf der Grundlage von 95 Expert*inneninterviews mit Vertreter*innen aller für die Entstehung deutscher Kinospielfilme relevanten Betätigungsfelder (Drehbuch, Regie, Produktion, Verleih, Kinoabspiel, Festivals, Förderung, öffentlich-rechtliches Fernsehen) sowie Dokumentenanalysen wird argumentiert, dass die filmische Aussagenentstehung unter dem Dach des Filmförderkomplexes neben dem Bemühen um Kunst, Wirtschaftlichkeit und Publikumswünschen auch eine politische Dimension besitzt und Machtverhältnisse in der Gesellschaft weiter verfestigt. Da spezifische Akteurskonstellationen und soziale Strukturen autonomes Handeln auf Seiten der Kommunikator*innen im Filmproduktionsprozess deutlich begrenzen und damit auch das Spektrum filmischer Wirklichkeitskonstruktionen einengen, können Kinospielfilme in Deutschland keine transformative Kraft entwickeln und das dem Medium theoretisch innewohnende emanzipatorische Potenzial kaum entfalten.

Thomas Wiedemann: Filmförderung als Transformationsbremse. Die politische Gestaltung der Kinospielfilmproduktion in Deutschland. In: Nils S. Borchers, Selma Güney, Uwe Krüger und Kerem Schamberger (Hrsg.): Transformation der Medien - Medien der Transformation. Verhandlungen des Netzwerks Kritische Kommunikationswissenschaft. Frankfurt am Main: Westend 2021. DOI: https://doi.org/10.53291/JHTJ5611.

Dr. Thomas Wiedemann I Ludwig-Maximilians-Universität München I thomas.wiedemann@ifkw.Imu.de 


\section{Erkenntnisinteresse und Relevanz}

Vor dem Hintergrund des Filmförderkomplexes beleuchtet dieser Beitrag kritisch die Mechanismen der Aussagenentstehung im Produktionsprozess von Kinospielfilmen in Deutschland. Gestützt auf den Ansatz der Akteur-Struktur-Dynamiken und mit einer Materialbasis von 95 Expert*inneneninterviews sowie Dokumentanalysen wird gezeigt, dass das heimische Filmschaffen auch eine politische Dimension besitzt und Machtverhältnisse in der Gesellschaft weiter verfestigt. Die nationale Kinospielfilmproduktion tut sich infolgedessen schwer, auf die großen Herausforderungen der Gegenwart (Krise der Demokratie, Klimawandel, Demographie, Migration, Digitalisierung, Arbeit im Spätkapitalismus und viele mehr) progressiv zu reagieren.

Den Ausgangspunkt bildet die Tatsache, dass Kinospielfilme gerade in Zeiten der audiovisuellen Durchdringung des Alltags sowie ihrer medienübergreifenden Präsenz eine Form der öffentlichen Kommunikation darstellen (Prommer 2016; Beck 2018), sich an ein Publikum richten und mit Funktionen verbunden werden oder, allgemeiner formuliert, in einem wechselseitigen Bedingungsverhältnis mit der Gesellschaft stehen (vgl. Heinze et al. 2012). Folgt man einer grundsätzlich konstruktivistischen Perspektive (vgl. Hepp et al. 2017), sind Filme kein Spiegel der Realität. Treffender ist, dass die von ihnen entworfenen Gesellschaftsbilder ein theoretisch unbegrenztes Wirklichkeitsspektrum mit eigenem Geltungsanspruch konstruieren und auf diesem Weg Wissen transportieren sowie Deutungsangebote und Sinnmuster zur Verfügung stellen (vgl. Schroer 2008; Mikos 2015). Denn als fiktionale Unterhaltungsangebote unterliegen sie zwar einem geringeren Faktizitätsanspruch als etwa journalistische Produkte (vgl. Stöber 2013, 170), doch können sie insbesondere aufgrund ihrer imaginativen Kraft, ihres Involvements und affektiven Potenzials eine strukturierende Wirkung entfalten, indem sie für Orientierung sorgen, die Integration fördern, der Herausbildung einer kollektiven Identität dienen und zur Erinnerungskultur beitragen sowie Antworten auf die drängendsten Fragen der Zeit geben (vgl. Mai 2006; Denzin 2010; Sutherland und Feltey 2013). Der Rolle von Kinospielfilmen als wirkmächtiges Referenzsystem für Pluralität und Diversität sowie als Motor gesellschaftlicher Emanzipation steht jedoch der Umstand gegenüber, dass sie von sozialen Faktoren geformt und somit durch die Gesellschaft vorstrukturiert sind (vgl. Silbermann 1980; Winter 2012). 
Bekannt ist, dass Kinospielfilme in einem arbeitsteiligen Komplex mit einer Vielzahl von Akteur*innen mit unterschiedlichen Zielhorizonten (beispielsweise Drehbuchautor*innen und Regisseur*innen an der kreativen Spitze gegenüber den für das Projektmanagement verantwortliche Produktionsunternehmen) entstehen und insofern sowohl eine kulturelle Praxis als auch ein Wirtschaftszweig sind (vgl. von Rimscha 2010; Grothe-Hammer 2015). Von der Forschung weniger berücksichtigt wurde bislang jedoch, dass die Kinospielfilmproduktion in Deutschland auch expliziter Gegenstand politischen Handelns ist. Dafür steht im Besonderen die öffentliche Filmförderarchitektur auf Bundes- und Länderebene, die jährlich fast eine halbe Milliarde Euro in die nationale Filmbranche steckt. ${ }^{1}$ Hinzu kommt, dass die große Mehrheit deutscher Kinospielfilme von öffentlichrechtlichen Rundfunkanstalten - Institutionen, die neben gesellschaftlichen auch politischen Einflüssen unterliegen - koproduziert wird (vgl. von Heinz 2012), was ebenso die Unabhängigkeit der Filmschaffenden, insbesondere der Kommunikator*innen ${ }^{2}$ im deutschen Kinospielfilm, wie auch die Staatsferne des Mediums insgesamt in Frage stellt. Demzufolge steht zur Vermutung, dass im heimischen Filmschaffen gesellschaftliche Hierarchien zum Ausdruck kommen (vgl. Mikos 2015) und hier ferner eine Auseinandersetzung um legitime - mehrheitlich anerkannte - Wirklichkeitskonstruktionen stattfindet (vgl. Dörner und Vogt 2012), so dass in letzter Konsequenz anstelle gesellschaftlicher Transformationsprozesse ohnehin schon dominante Deutungsangebote und Sinnmuster im Diskurs weiter befeuert werden.

1 Angesichts der massiven Beeinträchtigungen der Filmbranche im Zuge der Corona-Krise haben die Filmfördereinrichtungen aktuell darüber hinaus eine Reihe von Hilfsprogrammen gestartet. Die zur Verfügung gestellten Mittel belaufen sich insgesamt voraussichtlich auf rund 100 Millionen Euro (vgl. Filmförderungsanstalt 2020a).

2 Wie in der Kommunikationswissenschaft üblich sind mit Kommunikator*innen hier und im Folgenden die Handlungs- und Rollenträger*innen gemeint, die Aussagen öffentlicher Kommunikation bereitstellen, dabei schöpferisch und gestaltend tätig sind und so als Scharniere eine Schlüsselposition im Kommunikationsprozess einnehmen (vgl. Maletzke 1963, 43). Bezogen auf das Medium Film sind das insbesondere Akteur*innen aus den Bereichen Drehbuch, Regie und Produktion. 
Zur Ermittlung der Mechanismen und Konventionen, die das deutsche Filmschaffen in Anbetracht des politischen Gestaltungswillens prägen, fragt die vorliegende Studie mit Hilfe von Schimank, wie Akteurskonstellationen und soziale Strukturen die Aussagenentstehung deutscher Kinospielfilme in eine bestimmte Richtung lenken. Geleistet wird damit ein sozialwissenschaftlicher Beitrag zur Filmforschung, der in der Kommunikationswissenschaft anschlussfähig mindestens zur Kommunikatorforschung und zur Forschung im Bereich Medienpolitik ist. Welche Konstellationen von Akteur*innen sind im Produktionsprozess also von Bedeutung, wenn die Politik mitspricht? Wer hat die Möglichkeit, als Kommunikator*in an der Wirklichkeitskonstruktion im deutschen Kinospielfilm teilzuhaben und zu welchem Grad? Welche Anforderungen werden an die Kommunikator*innen im Produktionsprozess herangetragen? Welches Selbstverständnis zeichnet sie als Folge davon mehrheitlich aus und was sind ihre Strategien in der Interaktion mit anderen Akteur*innen? Zusammengefasst und kritisch gewendet: Sind Kinospielfilme in Deutschland angesichts dessen überhaupt in der Lage, als Segment der Gegenöffentlichkeit zu wirken, also denen eine Stimme zu geben, die sonst nicht gehört werden, und den hegemonialen Diskurs mit alternativen Wirklichkeitskonstruktionen herauszufordern?

Der folgende Abschnitt skizziert Schimanks Ansatz der Akteur-Struktur-Dynamiken, wendet ihn auf die soziale Konfiguration der deutschen Kinospielfilmproduktion an und informiert über Methode und Forschungsmaterial. Die Ergebnisse im dritten Abschnitt machen dann in vielerlei Hinsicht deutlich, dass das Filmschaffen in Deutschland alles andere als voraussetzungslos ist und "transformative Kinospielfilme« unter den Vorzeichen des Filmförderkomplexes nicht zu erwarten sind.

\section{Theorie, Methode und Forschungsmaterial}

Der Ansatz der Akteur-Struktur-Dynamiken, der die »wechselseitige Konstitution von handelndem Zusammenwirken und sozialen Strukturen « (Schimank 2007, 121-122) in den Mittelpunkt rückt und die Systemtheorie um eine Akteursperspektive erweitert, geht von (individuellen und kollektiven) Akteur*innen aus, die spezifische Interessen besitzen, über bestimmte (Macht-)Ressourcen verfügen und so eine Strategie für die Durchsetzung ihrer Ziele entwickeln (vgl. Schimank 2000). Dass sie mit ihrem Handeln stets auf andere Akteur*innen tref- 
fen, führt zu einer bewussten oder unbewussten Anpassung ihrer eigenen Handlungsstrategie - basierend auf ihren »Akteurfiktionen «, also ihrem Wissen über die Interessen und Ressourcen der anderen Akteur*innen sowie die Funktionslogik der sozialen Systeme, denen sie jeweils angehören (vgl. Schimank 1988). »Soziale Strukturdynamiken« werden somit »intentional vorangetrieben«, doch »entgleiten« sie den Akteur*innen »mal mehr, mal weniger ins Transintentionale (Schimank 2007, 125). Mit anderen Worten: Das Zustandekommen deutscher Kinospielfilme vollzieht sich aus dieser Perspektive in einer Aneinanderreihung von Konstellationen von Akteur*innen, die sich gegenseitig beobachten, beeinflussen und ihr Handeln notgedrungen aufeinander abstimmen. Und selbstverständlich gehören zu diesen Akteur*innen nicht nur die traditionell am Filmproduktionsprozess beteiligten Gewerke (von Drehbuch über Regie und Produktion bis hin zu Verleih und Kinoabspiel), sondern genauso die Filmförderung und das öffentlich-rechtliche Fernsehen mit ihren wirtschafts- und kulturpolitischen Zielsetzungen. Erst in solchen umfassenden Handlungsverkettungen können demzufolge filmische Konstruktionen von Realität erzeugt sowie soziale Strukturen erschaffen, erhalten und um- oder abgebaut werden.

Um das handelnde Zusammenwirken von Akteur*innen zu untersuchen, schlägt Schimank (2007) vor, die Konstellations-, Erwartungsund Deutungsstrukturen zu analysieren, in die es eingebettet ist und die es prägt. Für die Frage nach den Mechanismen und Konventionen der Aussagenentstehung im Produktionsprozess deutscher Kinospielfilme bedeutet das erstens, das »Können« der Kommunikator*innen im Filmschaffen hierzulande (Konstellationsstrukturen), also die eingespielten Gleichgewichte und Muster in ihrem Handeln (Herkunft, Sozialisation, Arbeitsbedingungen, Marktstrukturen, Ressourcen, Routinen, Zwänge), zu ergründen. Zweitens ist ihr »Sollen« zu ermitteln (Erwartungsstrukturen). Hier gilt es, die wesentlichen Ansprüche und Anforderungen herauszuarbeiten, mit denen die Kommunikator*innen im Filmproduktionsprozess konfrontiert werden (rechtliche Vorschriften, Richtlinien, formelle oder informelle Vorgaben der Filmförderung, Vorstellungen der Projektpartner einschließlich Fernsehen, Wünsche und Erwartungshaltungen der projektierten Zielgruppe). Drittens ist schließlich das »Wollen« der Kommunikator*innen zu adressieren (Deutungsstrukturen) und nach ihren evaluativen und kognitiven Orientierungen zu fragen (Selbstverständnis, Stoffauswahl und -umsetzung, Verhaltenskodex, Erfahrungen, Rezeptwissen). 
Gemäß dem hier vertretenen Verständnis von qualitativer theoriegeleiteter Forschung (vgl. Löblich 2016; Meyen et al. 2019) wurde auf Basis der theoretischen Perspektive ein Kategoriensystem mit den soeben skizzierten Konstellations-, Erwartungs- und Deutungsstrukturen als Hauptanalysedimensionen entwickelt. Wichtigstes Forschungsmaterial sind 95 Expert*inneninterviews mit Vertreter*innen aller für die Entstehung deutscher Kinospielfilme als zentral erachteten Betätigungsfelder. Das Sample orientierte sich dabei an der theoretischen Sättigung und umfasste zunächst sieben Drehbuchautor*innen, 35 Filmregisseur*innen (von denen etwa die Hälfte auch Drehbücher schreiben) sowie 19 Filmproduzent*innen, die sich nach Geschlecht, Alter, kulturellem Hintergrund und Ausbildung (Filmhochschulabsolvent*innen gegenüber Quereinsteiger*innen), aber auch mit Blick auf die Ausrichtung ihrer Arbeit (Mainstream gegenüber Arthouse) und ihren bisherigen Erfolg (künstlerisch gegenüber kommerziell) unterscheiden. Interviewt wurden außerdem sechs Vertreter*innen von großen und kleinen Verleihfirmen, sieben Kinobetreiber*innen (sowohl Multiplex als auch Programmkino) und fünf Filmfestival-Verantwortliche, ferner zwölf Angehörige nationaler und regionaler Filmfördereinrichtungen sowie vier Redakteur*innen öffentlich-rechtlicher Fernsehsender im Bereich Kinospielfilm. Um die so erzielten Erkenntnisse besser einschätzen zu können, waren die Expert*inneninterviews von mehreren Dutzend Dokumenten flankiert, darunter Marktdaten und Statistiken, Stellungnahmen von Branchenverbänden, offene Briefe, Thesenpapiere, Protokolle von Diskussionsveranstaltungen und journalistische Artikel.

\section{Ergebnisse}

Die Befunde der Studie können hier nur in Ausschnitten präsentiert werden. ${ }^{3}$ Nichtsdestotrotz veranschaulichen sie, dass der filmpolitische Gestaltungswille deutliche Spuren in den Konstellations-, Erwartungs- und Deutungsstrukturen des deutschen Filmschaffens hinterlässt und das Spektrum möglicher filmischer Wirklichkeitskonstruktionen in vielfacher Hinsicht einschränkt. Dem transformativen Potenzial von heimischen Kinospielfilmen wird so - willentlich oder nicht - ein Riegel vorgeschoben.

3 Für ausführlichere Befunde, wenngleich vorrangig bezogen auf das Gewerk Regie, vgl. Wiedemann (2018; 2019). 


\subsection{Konstellationsstrukturen}

Die Mehrheit der deutschen Kinospielfilmproduktionen sind Autor*innenfilme. Der Prozess der Wirklichkeitskonstruktion nimmt seinen Ausgang also in der Regel bei Drehbuchautor*innen und Regisseur*innen, die nicht selten auch an der Produktionsplanung und der Postproduktion teilhaben. Dazu gesellen sich gleichwohl meist schon während der Buchentwicklung Produzent*innen, die fortan bis zur Fertigstellung eines Films auch bei kreativen Entscheidungen mitsprechen und insofern, wenn auch in geringerem Maße, ebenso als Kommunikator*innen zu betrachten sind. Dennoch ist die Macht dieser letztgenannten Akteur*innen in Deutschland begrenzt. Es gibt kaum ein echtes Studiosystem und an den 157 deutschen Spielfilmen, die 2019 Kinopremiere hatten, waren knapp 200 Produktionsfirmen beteiligt (vgl. Spitzenorganisation der Filmwirtschaft 2020). Zu den Akteurskonstellationen im Produktionsprozess deutscher Kinospielfilme zählen ferner rund 120 Verleihfirmen, die im günstigsten Fall schon in der Entwicklungsphase eine Distributionszusage abgeben, sowie über 1600 Kinos als traditionell wichtigster kommerzieller Auswerter, wenngleich diese an vorderster Stelle von den gegenwärtigen Umbrüchen der Branche (Stichwort: Home Entertainment) betroffen sind. Zu nennen sind schließlich auch bedeutende deutsche Filmfestivals wie allen voran die Internationalen Filmfestspiele Berlin, auch bekannt als Berlinale, das Filmfest München, die Hofer Filmtage oder der Max-Ophüls-Preis in Saarbrücken, die längst eine zentrale Rolle für die Distribution und Zirkulation insbesondere von Arthouse-Filmen spielen, zumal sie für öffentliche Aufmerksamkeit sorgen und bisweilen weitere Einnahmequellen erschließen, zumindest wenn für die Film-Programmierung eine Bezahlung (»screening fees«) erfolgt.

Nichtsdestotrotz: Nur ein Viertel der durchschnittlichen Produktionskosten eines deutschen Kinospielfilms, die etwa drei bis vier Millionen Euro betragen, ist durch Eigenmittel, Rückstellungen und idealerweise Verleihgarantie und Weltvertrieb gedeckt. Rund die Hälfte des Budgets stammt dagegen aus den Töpfen der öffentlichen Filmfördereinrichtungen (darunter Filmförderungsanstalt, Deutscher Filmförderfonds, FilmFernsehFonds Bayern, Film- und Medienstiftung Nordrhein-Westfalen und Medienboard Berlin-Brandenburg), die 2019 ein Gesamtfördervolumen in Höhe 455,92 Millionen Euro auswiesen (vgl. Filmförderungsanstalt 2020b). Natürlich: Ohne diese umfassende Unterstützung kann der deutsche Film in seiner gegenwärtigen Form - gerade mit Blick auf sein hohes Produktionsaufkommen - nicht überleben. Aller- 
dings fungiert die Filmförderung auch als Gatekeeper, denn nur etwa jedes fünfte Filmprojekt erhält Förderung und kann realisiert werden: »Die Förderzusage für deinen Film bedeutet ganz klar das Ende eines anderen Filmprojekts«, kommentierte ein befragter Produzent. Dabei sind Verrenkungen in der Produktionsplanung und »Fördertourismus« (ein geförderter Film erhält meist nicht nur von einer Einrichtung Zuschüsse) auch im besten Fall einzukalkulieren, wie die interviewten Produzent*innen einstimmig erklärten. Für noch verbleibende Lücken im Budget (rund 25 Prozent) wird schließlich schon frühzeitig der Verkauf der TV-Lizenz angestrebt - in der Regel an öffentlich-rechtliche Fernsehsender, die im Gegenzug weitreichende Rechte in Sachen Koproduktion erwerben. Auch hier findet ein strikter Selektionsprozess statt. Den entsprechenden Senderredaktionen (für den Nachwuchs zum Beispiel »Das kleine Fernsehspiel« im ZDF) kommt also ebenfalls eine gewichtige Rolle in den Konstellationsstrukturen hinter der filmischen Wirklichkeitskonstruktion zu.

Ein Blick auf die Herkunft und Sozialisation der Kommunikator*innen zeigt, dass Drehbuchautor*innen, Regisseur*innen und Produzent*innen in Deutschland unabhängig von ihrem Alter fast ausnahmslos der oberen Mittelschicht entstammen und einen Familienhintergrund aufweisen, in der die Entscheidung für eine Tätigkeit in der Filmbranche ideell und finanziell Unterstützung erfährt. »Meine Eltern haben mich nicht unter Druck gesetzt, etwas Handfestes zu lernen«, resümierte stellvertretend ein Regisseur und berichtete von den »Finanzspritzen«, die er bis zu seinem 30. Lebensjahr von zu Hause bekam. Außerdem absolvierten die meisten befragten Kommunikator*innen ein Studium an der Filmhochschule, einer oft staatlichen und stark zugangsbeschränkten Ausbildungsstätte, dies es hierzulande nur an wenigen Standorten gibt, was die Elitisierung unter den Kommunikator*innen weiter verstärkt (vgl. Wiedemann 2019). Dort sammelt der Nachwuchs das nötige Startkapital für den Berufseintritt (insbesondere Social Skills, also Teamfähigkeit, Kompromissbereitschaft und die Wertschätzung von Teamarbeit, sowie Kontakte - zu Kommiliton*innen, noch wichtiger aber zu Förderreferent*innen und TV-Redakteur*innen, die zu Pitch-Terminen regelmäßig geladen werden), wird aber zugleich auch mit den Gesetzmäßigkeiten des Filmemachens hierzulande, vor allem den herrschenden Interaktionsroutinen, vertraut gemacht und so zu konformem Verhalten angeleitet. Ein ArthouseFilmemacher fasste es so zusammen: »Wir werden ausschließlich auf diesen kleinen Markt hin ausgebildet.« 
Die Ressourcen, die für eine Teilhabe an der filmischen Wirklichkeitskonstruktion in Deutschland erforderlich sind, verweisen einmal mehr auf das hohe Gewicht, das der Filmförderung und dem öffentlich-rechtlichen Fernsehen in den Akteurskonstellationen im Produktionsprozess deutscher Kinospielfilme zukommt. Deutlich zutage trat in den Interviews, dass die Kommunikator*innen für einen dauerhaften Verbleib in der Branche neben künstlerischem und technischem Know-how, Organisationstalent und finanzieller Absicherung mindestens ebenso Beziehungen und eine »Visitenkarte (ein Film mit Publikumserfolg, eine Auszeichnung) vorweisen müssen - die Währung schlechthin, um für den nächsten Film Fördermittel und Sendergelder zugesprochen zu bekommen. Ohne Zweifel ist die Zementierung von Diversitätsgrenzen auch dadurch vorprogrammiert. So treffen sozial Benachteiligte, nicht zu Kompromissen bereite Außenseiter, Einzelgänger*innen und allgemein Frauen (vgl. Loist und Prommer 2019), die allesamt aufgrund ihrer Herkunft und ihres Lebensweges vermutlich eher in der Lage sein dürften, den herrschenden Diskurs in Frage zu stellen und Räume für alternative Wirklichkeitskonstruktionen zu öffnen, auf deutlich gröBere Hindernisse, dauerhaft an der Aussagenentstehung im Produktionsprozess deutscher Kinospielfilme mitzuwirken.

\subsection{Erwartungsstrukturen}

Wenig überraschend treffen in den beschriebenen Akteurskonstellationen ganz unterschiedliche Zielsetzungen aufeinander, die als Erwartungsstrukturen Einfluss auf die Aussagenentstehung deutscher Kinospielfilme nehmen. Beschränkt man sich zunächst auf die traditionell am Filmproduktionsprozess beteiligten Akteur*innen, wird deutlich, dass die narrativen und ästhetischen Visionen der für Drehbuch und Regie Verantwortlichen notgedrungen mit dem Gebot der wirtschaftlichen Rentabilität konfrontiert werden. Dies bezieht sich auf Produzent*innen, die sich zwar als kreative Kompliz*innen verstehen, aber dennoch Risikoabwägungen treffen müssen. Entsprechend werden ihnen von den interviewten Regisseur*innen und Autor*innen oft Mutlosigkeit und Vorbehalte gegenüber provokanten Stoffen attestiert. Ähnliches trifft zu für Verleihfirmen, die unabhängig von ihrer Größe und Ausrichtung auf Gewinnmaximierung und Zielgruppenorientierung aus sind und umso mehr Mitsprache einfordern, je eher sie in ein Projekt eingebunden sind. »Wenn ich mit der Besetzung oder mit dem 
Film-Ende nicht einverstanden bin «, berichtete etwa selbstbewusst der Chef eines Independent-Verleihs, »dann gebe ich meiner kommerziellen Perspektive unmissverständlich Ausdruck.« Diese Praxis wurde von den Kommunikator*innen im deutschen Filmschaffen allerdings kaum in Frage gestellt, da sie Verleihfirmen als Sprachrohr der Kinobetreiber*innen erachten, welche die Wünsche des Publikums aus erster Hand erfahren und Experimente scheuen. »Wir sterben für Filmkunst. Aber am Ende des Tages müssen wir unsere Löhne bezahlen«, erklärte dazu der Betriebsleiter zweier Programmkinos in Frankfurt am Main, und die Betreiberin eines Münchner Kinos im Premiumsegment sagte: „Wir setzen auf Bewährtes. Egal ob es immer von denselben vier, fünf Leuten kommt.«

Natürlich spielt Rentabilität auch bei den Erwartungen der wichtigsten beiden Finanziers deutscher Kinospielfilme eine Rolle. Der Filmförderung und dem Fernsehen geht es aber nicht nur darum. Die Spielfilmredaktionen der öffentlich-rechtlichen Rundfunkanstalten, um hier zu beginnen, verweisen einerseits auf den im Rundfunkstaatsvertrag festgeschriebenen Kulturauftrag, betonen andererseits aber auch ihre Zuschauerorientierung und verknüpfen diese mit dem Anspruch auf Aktualität. »Unser Ziel«, so erklärte die Redakteurin einer Landesrundfunkanstalt der ARD, »ist ein Archiv der Gegenwart. Die von uns koproduzierten Filme sollen repräsentieren, was die deutsche Bevölkerung gerade bewegt: Flüchtlingskrise, Europa, aber auch Umwelt, Comingof-Age, Cybermobbing.« Ist ein Koproduktionsvertrag unterzeichnet, haben die verantwortlichen Redakteur*innen weitreichende Entscheidungsbefugnisse bei der Buchentwicklung und dem Cast, aber auch in Sachen Kamera und Postproduktion, wenngleich sie beteuern, vorrangig beratend tätig zu sein. Dennoch beurteilten die meisten interviewten Drehbuchautor*innen, Regisseur*innen und Produzent*innen ihre Zusammenarbeit mit den Sendern rückblickend als problematisch und monierten die verbreitete Angst, an Tabus zu rütteln oder von den gesellschaftlich relevanten Gruppen, welche die Sender in den Aufsichtsgremien kontrollieren, zur Rechenschaft gezogen zu werden. Ein weiterer Kritikpunkt ist die zunehmende Konvergenz von Kino- und Fernsehfilmen (vgl. Mikos 2011) zugunsten der Fernsehlogik, für die Alt-Filmemacher Edgar Reitz in einem Thesenpapier anlässlich des Kongresses »Zukunft deutscher Film« zu Perspektiven der deutschen Kino- und Filmkultur 2018 in Frankfurt am Main deutliche Worte fand: Da sich »halbstaatliche Superproduzenten« in alle Geschmacksentscheidungen einmischten, erkenne man den »camouflierten Fernseh- 
film [...] sofort an seiner thematischen Überspanntheit und der Instrumentalisierung der Handlungsfiguren« (Reitz 2018).

Das oberste Ziel der Filmförderinstitutionen ist einerseits die wirtschaftliche Stärkung der heimischen Filmindustrie, weshalb Wert gelegt wird auf Standorteffekte und kommerziell erfolgversprechende Produktionen, die dem System seinerseits Einnahmen bescheren, sei es in Form der Rückzahlung von Darlehen oder, im Fall der Filmförderungsanstalt, über die sogenannte Filmabgabe, die Kinos jährlich zu entrichten haben. Andererseits möchte man als Beitrag zu einer lebendigen deutschen Filmkultur künstlerisch herausragende Projekte unterstützen, die dann auf dem (internationalen) Filmfestival-Parcours reüssieren oder Auszeichnungen wie den Deutschen Filmpreis erhalten sollen. Folgt man Monika Grütters, Beauftragte des Bundes für Kultur und Medien, zielt die kulturelle Filmförderung (insbesondere gebündelt bei der Staatsministerin selbst) auf anspruchsvolle Arthouse-Filme und innovative Formate, denn »nur so entsteht Fortschritt, nur so, mit kritischen Positionen, halten wir unsere Demokratie wach « (zitiert nach Kniebe 2019). Abgesehen von der automatischen Förderung für Filmvorhaben, die mindestens ein Viertel ihrer Kosten in Deutschland auszugeben beabsichtigen, seitens des Deutschen Filmförderfonds und der Referenzfilmförderung (bemessen am kommerziellen oder künstlerischen Erfolg des Vorgängerfilms) befindet über die selektive Projektfilmförderung meist eine aus der Branche rekrutierte Expert*innenkommission mit Zweidrittelmehrheit hinter verschlossenen Türen. Entscheidungsgrundlage sind, wie die interviewten Kommissionsmitglieder angaben, das Drehbuch und das »kreative Package«, also die Projektbeteiligten und ihre Reputation (die Kooperation mit einem Sender ist ebenfalls mindestens gerne gesehen), sowie die Produktionsparameter einschließlich des zu erwartenden Standortnutzens.

Auch hier gibt es Kritik auf Seiten der Filmschaffenden - wegen der zu sehr auf Regionaleffekte bedachten Länderförderungen (laut einer Autorenfilmemacherin drohe das »Menetekel: Schreibt das Drehbuch doch um auf Berlin«), vor allem aber aufgrund der Konsensorientierung in den Fördergremien. So würden, folgt man einem Regisseur mit jahrzehntelanger Berufserfahrung, die Vorstellungen in den Gremien immer »konservativer, ängstlicher und kulturspießiger «, so dass nur noch Verantwortung übernommen werde für Feel-Good-Komödien und den »Problemfilm, der gemacht werden muss und zwar möglichst konventionell«. Wenig anders hält das von vielen namhaften Filmschaffenden erarbeitete Abschlusspapier des oben erwähnten Kon- 
gresses zu Perspektiven der deutschen Kino- und Filmkultur fest, dass es dem sogenannten »Gremienfilm« an »Ecken und Kanten« fehle und nur das durchgewinkt werde, was von »etablierten« Filmemacher*innen komme oder an »Bestehendes« anknüpfe. Für »das Extreme, das Ungewöhnliche, das Bodenlose und das ästhetisch Innovative« fließe dagegen kein Geld (Frankfurter Positionen 2018, 3). In der Tat können die wichtigsten deutschen Filmfestivals hier nur auf dem Papier als Gegengewicht erachtet werden. Denn insbesondere Deutschlands A-Filmfestival, die Berlinale, orientiert sich als staatlich bezuschusstes Flaggschiff des nationalen Films in seiner Ausrichtung und Programmpolitik vorrangig an dieser wirtschafts- und kulturpolitischen Agenda (vgl. Wiedemann und Krainhöfer 2018).

\subsection{Deutungsstrukturen}

Die für den Filmproduktionsprozess in Deutschland typischen Akteurskonstellationen ziehen nicht nur eine Reihe von Forderungen und Ansprüchen nach sich, wie die Wirklichkeitskonstruktion deutscher Kinospielfilme beschaffen sein soll. Der hohe Stellenwert der Förderung und des öffentlich-rechtlichen Fernsehens sowie die daraus resultierenden Erwartungen an das Filmschaffen hierzulande schlagen sich, in Verbindung mit generellen Machtstrukturen in der Gesellschaft, auch auf die evaluativen und kognitiven Orientierungen der Kommunikator*innen im deutschen Kinospielfilm nieder. Solche Deutungsstrukturen beziehen sich zunächst auf eine klar erkennbare Berufsideologie. Dazu gehören - diese Begriffe tauchen in den Interviews immer wieder auf die Erfahrung von »Durststrecken« und »Niederlagen « zu Karrierebeginn, gepaart einerseits mit »Dankbarkeit«, andererseits aber auch mit "Stolz«, es schließlich geschafft, also gegenüber Mitstreitenden mehr »Durchsetzungsvermögen « an den Tag gelegt zu haben und dabei immer »stur« der »inneren Stimme gefolgt« zu sein, ohne den »eigenen Kopf « aufgegeben zu haben.

Das Selbstverständnis der Kommunikator*innen gestaltet sich dann recht homogen. Während nur wenige Autorenfilmemacher*innen erklärten, sie wollten die Zuschauer*innen nicht nötigen, »etwas Bestimmtes zu denken«, und verfolgten »keinen pädagogischen oder didaktischen Ansatz«, versteht sich das Gros der Interviewten als Themensetzer*innen und Aufklärer*innen über "gesellschaftlich relevante Sachverhalte« (Regisseur). Das Ansinnen, den »Unterhaltungscharak- 
ter von Filmen« mit einem »ernsthaften Diskurs« zu verbinden (Drehbuchautor), ist dementsprechend die Regel. Von einem Produzenten treffend formuliert: »Es macht mich sehr glücklich, mit diesem brisanten Thema auf dem Höhepunkt der Flüchtlingsdebatte so viele Leute erreicht zu haben.«Doch, wie gleich noch deutlicher wird, nehmen die Kommunikator*innen ihre Themensetzer*innen- und Aufklärer*innenrolle - den »Akteurfiktionen « entsprechend - lediglich in einem eng abgesteckten Rahmen wahr.

Das Wissen um die Ziele und Ressourcen der anderen Akteur*innen macht sich darüber hinaus in einer gehörigen Portion Pragmatismus bemerkbar. Neben dem oftmaligen »Kuhhandel« um Szenen (Drehbuchautor) zwischen den Kommunikator*innen selbst bedeutet das beispielsweise, dass nach dem Verleiher-Feedback, ohne mit der Wimper zu zucken, »kooperativ« (Drehbuchautorin und Regisseurin) neue Buch- oder Schnittfassungen erstellt werden. Ebenso sind von der Stoffentwicklung bis zur Postproduktion Zugeständnisse an die Vorstellungen der beteiligten Fernsehredakteur*innen an der Tagesordnung. »Mein Film«, so erinnerte sich eine Autorenfilmemacherin an ihr Kinodebüt, »wurde durch den WDR-Redakteur sehr stark in Richtung Realismus gedrängt.« Dass die Filmförderung auf die tatsächliche Produktion kaum noch Einfluss nimmt, ändert nicht viel, denn die Erwartungshaltung dieses Akteurs wurde von den Kommunikator*innen schon längst - wieder gemäß Schimanks Vorstellung von »Akteurfiktionen « - antizipiert. Man sei »beim Schreiben und Entwickeln in den Gesetzmäßigkeiten der Branche gefangen «, offenbarte ein Drehbuchautor, der mittlerweile auch als Produzent tätig ist. Man wisse, »was man tun kann, um Geld zu bekommen «, und erfülle so Erwartungen, »ohne dass diese überhaupt bewusst formuliert sein müssen«.

Mehr noch: Dass selbst etablierte Filmemacher*innen und Produzent*innen wiederholt von Konflikten berichteten und ihre Kompromissfähigkeit schließlich als Tugend deklarierten, unterstreicht, wie begrenzt die Autonomie der Kommunikator*innen im deutschen Filmschaffen ist. »Es gibt viele Sachen, die ich gerne erzählen würde. Aber die will keiner hören«, gestand eine Drehbuchautorin und ist damit keine Ausnahme. Stattdessen vollzieht sich die Aussagenentstehung deutscher Kinospielfilme fast ausschließlich unter strenger Einhaltung folgender Kriterien: die Grenze zwischen Arthouse und Mainstream wahren, keine Genre-Experimente wagen, sondern lieber beispielsweise auf Culture-Clash- und Family-Entertainment-Formate oder historische Stoffe (am besten Biographien) setzen, sowie Themenfilme 
»geschmackvoll« und »ernst« umsetzen. Dass dabei die Systemfrage gestellt wird, ist auch angesichts der Herkunft und Sozialisation der meisten Kommunikator*innen alles andere als wahrscheinlich.

\section{Fazit}

Gestützt auf den Ansatz der Akteur-Struktur-Dynamiken zeigt die Untersuchung der dominanten Konstellations-, Erwartungs- und Deutungsstrukturen im Produktionsprozess deutscher Kinospielfilme, dass sich das heimische Filmschaffen keineswegs bloß im Spannungsfeld zwischen Kunst, Wirtschaftlichkeit und Publikumswünschen bewegt. Vielmehr weist die Kinospielfilmproduktion in Deutschland, das belegen die Ergebnisse in vielfacher Hinsicht, auch eine politische Dimension auf und zeichnet sich in der Tat durch klare Hierarchien aus, was sich in den filmischen Deutungsangeboten und Sinnmustern niederschlägt und schlussendlich die bestehenden Machtverhältnisse in der Gesellschaft weiter verfestigen dürfte. Denn als Kommunikator*innen nehmen vor allem Drehbuchautor*innen und Regisseur*innen zwar eine Schlüsselposition für die Wirklichkeitskonstruktion von Kinospielfilmen ein, doch übernehmen den Gegenpart im Kampf zwischen Kreativen und Geldgebern keineswegs nur Produktionsunternehmen, Verleihfirmen und Kinoauswerter, sondern mindestens ebenso die Filmförderung und das öffentlich-rechtliche Fernsehen als wichtigster Finanzier oder Koproduktionspartner. Die Folge: Kinospielfilme können in Deutschland erst realisiert werden und ein Publikum finden, wenn die Filmförderung von der Lukrativität oder Relevanz des Vorhabens überzeugt ist und der Stoff zum Profil der öffentlich-rechtlichen Sender passt.

Mehr noch: Neben der Elitisierung der Branche (und etwa auch der Zementierung von Geschlechterungerechtigkeiten) sowie der Herausbildung einer klaren Berufsideologie auf Seiten der Kommunikator*innen versuchen insbesondere die Filmemacher*innen, gemäß ihren »Akteurfiktionen « an Bestehendes anzudocken. Der wesentliche Grund hierfür ist die Konstellation mit weiteren Akteur*innen im Filmproduktionsprozess, die über Risikomanagement, Gewinnmaximierung und Zielgruppenorientierung nachdenken, vor allem jedoch Vorbehalte gegenüber provokanten Stoffen haben, auf Publikumserfolge hoffen und sich mit Auszeichnungen schmücken möchten sowie eine standort- und kulturpolitische Agenda verfolgen. Während »gefördert wird, 
was Deutschland braucht«, so formulierte es Regisseur Dominik Graf bereits 2012 in der Zeit (Graf 2012), gelingt es mit dem Filmförderkomplex offenbar nicht, Diversität und Pluralität zu verwirklichen. In diesem Sinne: Ohne eine strukturelle Reform des Filmfördersystems, wie sie im Übrigen auch von einigen Brancheninitiativen propagiert wird (neben den genannten Frankfurter Positionen beispielsweise auch von ProQuote Film und vom Hauptverband Cinephilie), lassen sich die imaginative Kraft und das emanzipatorische Potenzial von Spielfilmen allenfalls bedingt ausschöpfen. Dass von der nationalen Kinospielfilmproduktion Impulse bezogen auf die großen Herausforderungen der Gesellschaft ausgehen oder gar Transformationsprozesse aktiv vorangetrieben werden, ist demzufolge nicht zu erwarten.

\section{Literatur}

Beck, Klaus. 2018. Das Mediensystem Deutschlands. Strukturen, Märkte, Regulierung. Wiesbaden: Springer VS.

Denzin, Norman K. 2010. Reading Film. Filme und Videos als sozialwissenschaftliches Erfahrungsmaterial. In: Qualitative Forschung. Ein Handbuch, herausgegeben von Uwe Flick, Ernst von Kardoff und Ines Steinke, 416-428. Reinbek bei Hamburg: Rowohlt.

Dörner, Andreas, und Ludgera Vogt. 2012. Unterhaltungskultur als politische Kultur. Politikvermittlung in der Gegenwartsgesellschaft. In: Unterhaltungsrepublik Deutschland. Medien, Politik und Entertainment, herausgegeben von Andreas Dörner und Ludgera Vogt, 11-31. Bonn: Bundeszentrale für politische Bildung.

Filmförderungsanstalt 2020a. Soforthilfeprogramme der Filmförderungen. https://www.ffa.de/corona-soforthilfe-1.html. Zugegriffen: 29. September 2020.

Filmförderungsanstalt. 2020b. Zahlen aus der Filmwirtschaft. https://www.ffa. de/download.php?f=5d26f0e1995b98c331af13c33ff775aa\&target $=0$. Zugegriffen: 23. August 2020.

Frankfurter Positionen. 2018. Zur Zukunft des deutschen Films. www.lichter-filmfest.de/media/180426_zdf_ergebnisspapier_06.pdf. Zugegriffen: 27. August 2020.

Graf, Dominik. 2012. Das Grauen ... das Grauen! Die Zeit vom 26. April, https:// www.zeit.de/2012/18/Deutscher-Filmpreis. Zugegriffen: 28. August 2020.

Grothe-Hammer, Michael. 2015. »Du passt auf, dass der Irre pünktlich fertig wird.« Zum Machtgleichgewicht zwischen Regie und Produktionsleitung in Filmprojekten. Studies in Communication and Media 4 (3): 189-247.

Heinz, Julia von. 2012. Die freundliche Übernahme. Der Einfluss des öffentlich-rechtlichen Fernsehens auf den deutschen Kinofilm von 1950 bis 2010. Baden-Baden: Nomos. 
Heinze, Carsten, Stephan Moebius, und Dieter Reicher (Hrsg.). 2012. Perspektiven der Filmsoziologie. Konstanz: UVK.

Hepp, Andreas, Wiebke Loosen, Uwe Hasebrink, und Jo Reichertz. 2017. Konstruktivismus in der Kommunikationswissenschaft. Über die Notwendigkeit einer (erneuten) Debatte. Medien \& Kommunikationswissenschaft 65 (2): 181-206.

Kniebe, Tobias. 2019. »Das Geld wird sinnvoll genützt.« Interview mit Monika Grütters. Süddeutsche Zeitung vom 31. Juli, 9.

Löblich, Maria. 2016. Theoriegeleitete Forschung in der Kommunikationswissenschaft. In: Handbuch nicht standardisierte Methoden in der Kommunikationswissenschaft, herausgegeben von Stefanie Averbeck-Lietz und Michael Meyen, 6779. Wiesbaden: Springer VS.

Loist, Skadi, und Elizabeth Prommer, 2019. Gendered production culture in the German film industry. Media Industries 6 (1): 95-115.

Mai, Manfred, 2006. Künstlerische Autonomie und soziokulturelle Einbindung. Das Verhältnis von Film und Gesellschaft. In: Das Kino der Gesellschaft - die Gesellschaft des Kinos. Interdisziplinäre Positionen, Analysen und Zugänge, herausgegeben von Manfred Mai und Rainer Winter, 24-47. Köln: Herbert von Halem.

Maletzke, Gerhard. 1963. Psychologie der Massenkommunikation. Theorie und Systematik. Hamburg: Hans-Bredow-Institut.

Meyen, Michael, Maria Löblich, Senta Pfaff-Rüdiger, und Claudia Riesmeyer. 2019. Qualitative Forschung in der Kommunikationswissenschaft. Eine praxisorientierte Einführung. Wiesbaden: Springer VS.

Mikos, Lothar. 2011. Amphibischer Film versus transmediale Erzählung. Zu den komplexen Wechselbeziehungen zwischen Film und Fernsehen. In: Kino in Bewegung. Perspektiven des deutschen Gegenwartsfilms, herausgegeben von Thomas Schick und Tobias Ebbrecht, 137-154. Wiesbaden: VS Verlag für Sozialwissenschaften.

Mikos, Lothar. 2015. Film- und Fernsehanalyse. Konstanz: UVK.

Prommer, Elizabeth. 2016. Film und Kino. Die Faszination der laufenden Bilder. Wiesbaden: Springer VS.

Reitz, Edgar. 2018. Vier Thesen zur Erneuerung der Filmkultur. Unveröffentlichtes Manuskript.

Rimscha, Bjørn von. 2010. Risikomanagement in der Entwicklung und Produktion von Spielfilmen. Wie Produzenten vor Drehbeginn Projektrisiken steuern. Wiesbaden: VS Verlag für Sozialwissenschaften.

Schimank, Uwe. 1988. Gesellschaftliche Teilsysteme als Akteurfiktionen. Kölner Zeitschrift für Soziologie und Sozialpsychologie 40 (4): 619-639.

Schimank, Uwe. 2000. Handeln und Strukturen. Einführung in die akteurtheoretische Soziologie. Weinheim: Juventa.

Schimank, Uwe. 2007. Handeln in Konstellationen. Die reflexive Konstitution von handelndem Zusammenwirken und sozialen Strukturen. In: Journalismustheorie: Next Generation. Soziologische Grundlegung und theoretische Innovation, herausgegeben von Klaus-Dieter Altmeppen, Thomas Hanitzsch und Carsten Schlüter, 121-137. Wiesbaden: VS Verlag für Sozialwissenschaften. 
Schroer, Markus. 2008. Einleitung: Die Soziologie und der Film. In: Gesellschaft im Film, herausgegeben von Markus Schroer, 7-13. Konstanz: UVK.

Silbermann, Alphons. 1980. Zur soziologischen und sozialpsychologischen Analyse des Films. In: Filmanalyse. Grundlagen, Methoden, Didaktik, herausgegeben von Alphons Silbermann, Michael Schaaf und Gerhard Adam, 13-32. München: Oldenbourg.

Spitzenorganisation der Filmwirtschaft. 2020. Filmstatistisches Jahrbuch. BadenBaden: Nomos.

Stöber, Rudolf. 2013. Neue Medien. Geschichte. Von Gutenberg bis Apple und Google. Medieninnovation und Evolution. Bremen: Edition lumière.

Sutherland, Jean-Anne, und Kathryn Feltey (Hrsg.). 2013. Cinematic sociology: Social life in film. Thousand Oaks: Sage.

Wiedemann, Thomas. 2018. Die Logik des Filmemachens. Zwölf Interviews mit deutschen Filmregisseurinnnen und -regisseuren. Köln: Herbert von Halem.

Wiedemann, Thomas. 2019. Filmregisseurinnen und Filmregisseure in Deutschland. Strukturen und Logik eines heteronomen Berufsfeldes. Publizistik 64 (2): 205-223.

Wiedemann, Thomas, und Tanja C. Krainhöfer. 2018. Filmfestival dekonstruiert. Akteurskonstellationen in der Debatte zur Zukunft der Berlinale. Navigationen 18 (2): 177-196.

Winter, Rainer. 2012. Das postmoderne Hollywoodkino und die kulturelle Politik der Gegenwart. Filmanalyse als kritische Gesellschaftsanalyse. In: Perspektiven der Filmsoziologie, herausgegeben von Carsten Heinze, Stephan Moebius und Dieter Reicher, 41-59. Konstanz: UVK.

\section{Open Access}

Dieser Beitrag erscheint unter der Creative-Commons-Lizenz CC BY-ND 3.0 DE: https://creativecommons.org/licenses/by-nd/3.0/de/. 